\title{
Commentary \\ The evidence for small-volume resuscitation with hyperoncotic albumin in critical illness
}

John A Myburgh $1,2,3$

\author{
1Division of Critical Care and Trauma, The George Institute for International Health, Sydney, 2050, Australia \\ ${ }^{2}$ Faculty of Medicine, University of New South Wales, Sydney, 2000, Australia \\ ${ }^{3}$ Department of Intensive Care Medicine, St George Hospital, Sydney 2217, Australia
}

Corresponding author: John Myburgh, j.myburgh@george.org.au

Published: 30 April 2008

This article is online at http://ccforum.com/content/12/2/143

(c) 2008 BioMed Central Ltd

See related research by Jacob et al., http://ccforum.com/content/12/2/R34
Critical Care 2008, 12:143 (doi:10.1186/cc6882)

\begin{abstract}
Small-volume resuscitation of critically ill patients with hyperoncotic albumin offers a number of theoretical advantages, such as increasing intravascular volume in excess of the volume of fluid administered and reducing interstitial edema. Whilst iso-oncotic albumin has been shown to be equi-effective to isotonic saline for the resuscitation of critically ill patients without associated traumatic brain injury, the efficacy of hyperoncotic albumin for resuscitation has not been evaluated in large-scale randomizedcontrolled trials. Overall, the evidence for resuscitation with hyperoncotic albumin is limited by studies of poor methodological quality with heterogenous study populations and control regimens. There is marginal qualitative evidence of improvements in surrogate outcomes in disparate patient populations, but no evidence of any survival benefit associated with resuscitation with hyperoncotic albumin. Given the lack of evidence and clinical uncertainty about the efficacy of hyperoncotic albumin, a large-scale randomizedcontrolled trial is required to determine its role in the acute resuscitation of hypovolemic or hypoalbuminemic critically ill patients.
\end{abstract}

Resuscitation of critically ill patients with hyperoncotic fluids offers a number of theoretical advantages. These are presented in the systemic review by Jacob and colleagues in this issue of Critical Care [1]. Based on the principles described by Starling in 1896 [2], intravenous administration of hyperoncotic fluids under physiological conditions produces transient hypervolemia that may be augmented by intravasation of interstitial fluid into the vascular compartment. This may result in increased effective intravascular volume that exceeds the volume of administered fluid and may reduce pathological interstitial edema. Under time-critical conditions such as traumatic brain injury, burns or severe sepsis, resuscitation with small volumes of hyperoncotic fluid appear attractive, particularly in the pre-hospital setting where prompt delivery of smaller volumes of resuscitation solutions may be more effectively administered.
Hypertonic crystalloids, such as saline, have been advocated for small-volume resuscitation, particularly in the pre-hospital setting. However, a double-blind randomized-controlled trial comparing rapid infusion of $250 \mathrm{ml}$ of $7.5 \%$ hypertonic saline to Ringer's lactate to patients with severe traumatic brain injury did not demonstrate a difference in mortality or sixmonth neurological outcomes [3].

Of the hyperoncotic colloids, albumin has been used for resuscitation since the 1940 s, primarily for acute resuscitation in hypovolemic patients, but also for 'slow' resuscitation in hypoalbuminemic patients with liver disease undergoing abdominal paracentesis. Despite limited evidence for the use of hyperoncotic albumin in these contexts, unequivocal evidence for the efficacy of iso-oncotic (4\%) albumin for resuscitation of patients in the intensive care unit now exists following the publication of the Saline versus Albumin Fluid Evaluation (SAFE) study [4]. This study was prompted by the publication of a meta-analysis of 1,104 patients from 24 studies that concluded that the use of albumin was associated with an overall excess mortality of 6\% [5]. The SAFE study was a 6,997-patient, double-blind, prospective, randomizedcontrolled trial that demonstrated no difference in all-cause 28-day mortality between patients who received albumin or saline for intravenous resuscitation in the intensive care unit. In addition to providing evidence of safety for the use of $4 \%$ albumin for resuscitation in a heterogenous population of patients, the SAFE study also provided some insights into the effects of albumin in two important patient populations.

An additional analysis of 460 patients with traumatic brain injury from the SAFE study (the SAFE-TBI study) demonstrated that resuscitation with albumin was associated with significantly higher two-year mortality compared to saline 
(33.2\% versus $20.4 \%$, relative risk (RR) $1.63,95 \%$ confidence intervals $(\mathrm{Cl}) 1.17$ to $2.26, p=0.003$ ) [6]. These data provide compelling evidence for the selection of fluids or resuscitation of patients with traumatic brain injury and suggest that iso-oncotic albumin and, by inference, hyperoncotic albumin, be avoided in these patients as it is unlikely that such a study would be repeated.

Secondly, in 1,218 patients presenting with sepsis at baseline, resuscitation with albumin was associated with a relative risk of death of $0.87(95 \% \mathrm{Cl} 0.74$ to 1.02$)$ compared to saline, suggesting a potentially beneficial effect in sepsis that requires further study.

However, extrapolation of the results of the SAFE study to acute and 'slow' resuscitation with hyperoncotic colloids requires caution. In this regard, the systematic review about the efficacy of small-volume resuscitation with hyperoncotic albumin solutions by Jacob and colleagues [1] is timely.

This review identified 25 randomized-controlled trials with a total of 1,485 patients and is remarkably similar to the 1998 Cochrane Injuries Group Albumin Reviewers meta-analysis in terms of evaluable studies, patient numbers and variability of patient populations [5]. In contrast, Jacob and colleagues used a qualitative summary of surrogate outcome measures to determine the effects of hyperoncotic albumin on clinically relevant endpoints such as morbidity, organ function, length of stay and cost. A secondary quantitative meta-analysis on the effects on survival was also performed on studies where deaths were recorded on an intention-to-treat basis.

Overall, the quality of studies analyzed was poor, with a median number of patients per trial of 30 (interquartile range (IQR) 18 to 58) and only 3 of the 25 studies were conducted under blinded conditions. A range of control regimens were identified, the most common being comparisons with hydroxyethyl starch in nine trials. Similarly, there was a range of clinical conditions where hyperoncotic albumin was studied, the most common of which was sepsis (328 patients from 7 trials, 6 of which were from one investigator), liver disease (272 patients from 3 trials), neonates (81 patients from 3 trials) and one study of 18 patients with traumatic brain injury.

Despite these limitations, Jacob and colleagues conclude that on the basis of qualitative analyses of a range of surrogate outcome measures, the randomized-controlled trials included in the analysis suggest limited evidence of clinical benefit, such as reduction in morbidity in high-risk neonates and brain injury, avoidance of major organ edema in surgery and high-risk neonates, and preservation of renal function in surgery and liver disease. With respect to survival data, pooled data of 1,287 patients from 20 evaluable trials, for which the median duration of follow-up was 5 days (IQR 5 to 13 days), demonstrated no difference between hyperoncotic albumin and 'control' (RR $0.95,95 \% \mathrm{Cl} 0.78$ to
1.17). Furthermore, survival data from the patients with sepsis were opposite to the trend observed in patients with sepsis in the SAFE study.

The strength of the conclusions from the qualitative analysis of the included trials requires great caution given the methodological limitations and heterogeneity of trials. The use of outcomes subject to ascertainment bias, particularly in unblinded studies or non-blinded outcome adjudication and the use of surrogate outcome measure significantly question the validity of these conclusions [7].

Accordingly, clinicians should not change practice on the basis of this systematic review. Furthermore, the overall lack of improved efficacy between hyperoncotic albumin and control regimens demonstrated in the quantitative metaanalysis tends to affirm the view of a revised Cochrane metaanalysis that concluded that the use of colloid solutions for acute resuscitation of hypovolemic patients outside the context of randomized-controlled trials is difficult to justify [8].

This systematic review provides a useful summary of the limited published evidence for the use of hyperoncotic albumin for small-volume resuscitation. Clinical uncertainty about the efficacy of its use remains, apart from patients with traumatic brain injury where, on the basis of the SAFE-TBI study, use of albumin for resuscitation is contraindicated [6].

The imperative following this systematic review is to design and conduct a large-scale, double-blind, randomized-controlled trial to address the efficacy and cost-effectiveness of small-volume hyperoncotic albumin resuscitation in critically ill patients.

\section{Competing interests}

The author declares that they have no competing interests.

\section{References}

1. Jacob M, Chappell D, Conzen P, Wilkes MM, Becker BF, Rehm $\mathrm{M}$ : Small-volume resuscitation with hyperoncotic albumin: a systematic review of randomized clinical trials. Crit Care 2008, 12:R34.

2. Starling $\mathrm{EH}$ : On the absorption of fluids from connective tissue spaces. J Physiol 1896, 19:312-326.

3. Cooper DJ, Myles PS, McDermott FT, Murray LJ, Laidlaw J, Cooper G, Tremayne AB, Bernard SS, Ponsford J; HTS Study Investigators: Prehospital hypertonic saline resuscitation of patients with hypotension and severe traumatic brain injury: a randomized controlled trial. JAMA 2004, 291:1350-1357.

4. SAFE Study Investigators: A comparison of albumin and saline for fluid resuscitation in the intensive care unit. $N$ Engl J Med 2004, 350:2247-2256.

5. Cochrane Injuries Group Albumin Reviewers: Human albumin administration in critically ill patients: systematic review of randomised controlled trials. BMJ 1998, 317:235-240.

6. SAFE Study Investigators: Saline or albumin for fluid resuscitation in patients with traumatic brain injury. N Engl J Med 2007, 357:874-884.

7. Wager E, Tumas JA, Field EA, Glazer NB, Schulz G, Grossman L: Improving the conduct and reporting of clinical trials. JAMA 2000, 283:2788-2789.

8. Perel P, Roberts I: Colloids versus crystalloids for fluid resuscitation in critically ill patients. Cochrane Database Syst Rev 2007, CD000567. 